WE1E-1

\title{
RECENT PROGRESS IN U.S. MILLIMETER WAVE SPECTRUM MANAGEMENT POLICY
}

\author{
Michael J. Marcus \\ Office of Engineering and Technology (M/S 1300) \\ Federal Communications Commission \\ Washington, DC 20554 \\ e-mail: mmarcus@fcc.gov
}

\begin{abstract}
This paper describes recent actions by the Federal Communications Commission to establish routine rules for the commercial use of frequencies above $40 \mathrm{GHz}$. These rules will enable the development and sale of new products in this spectrum which will pioneer the commercial use of the millimeter wave region.
\end{abstract}

\section{INTRODUCTION}

While millimeter wave technology has been used and is being proposed for a variety of military systems, its use in commercial systems has been limited to date. In the U.S. the only significant published research on commercial systems deals with automotive radar systems. Major reasons for this lack of commercial use include the cost of such technology to date and the availability of lower spectrum with more conventional propagation characteristics. The availability of new technology, such as monolithic microwave integrated circuits (MMIC), resulting from military research and development, crowding of lower spectrum, better understanding of the nature and implications of millimeter wave propagation, and the marked decrease in military electronics spending have resulted in increased commercial interest in this area.
In addition, some other countries' spectrum management administrations have been explicitly urging their industries to pursue such technology for a variety of reasons including international competitiveness.

A major difference between radio technology and other electronics technology is the role of government policy in spectrum management. While computer manufacturers, for example, do not need to get nonroutine government approval for computers with breakthrough technology, new types of radio technology do require national spectrum management approval before they can be marketed in most countries. This approval may be subject to both national and international considerations. This paper deals with recent actions by the Federal Communications Commission (FCC) to establish rules for commercial use of frequencies above 40 $\mathrm{GHz}$.

\section{PROPOSALS}

On October 20, 1994 the FCC formally proposed various rules for commercial use of frequencies above $40 \mathrm{GHz}^{1}$. The proposals dealt with 13 bands between 40 and 153 $\mathrm{GHz}$. Some of the proposed bands would have been unlicensed, while others would 
have been licensed in conjunction with an auction. In response to these proposals, comments were received from 44 parties and reply comments were received from a similar number ${ }^{2}$. The main interests expressed in the comments dealt with unlicensed use of 59-64 GHz and vehicular radar in two other bands.

\section{DECEMBER 1995 DECISIONS}

Guided by the comments, the FCC on December 15, 1995 adopted an initial set of millimeter wave rules and made several more proposals ${ }^{3}$. 46.7-46.9 GHz and 76-77 $\mathrm{GHz}$ were made available for unlicensed vehicular radars. 59-64 $\mathrm{GHz}$ was made available for unlicensed use by any application except vehicular radars.

In the case of vehicular radars there are several power limits for the transmitters. Some of these were motivated by concerns about exposure to individuals by the RF power. When the vehicle is not in motion, the power density may not exceed 100 $\mathrm{nW} / \mathrm{cm}^{2}$ at $3 \mathrm{~m}$ from the surface of the radiating structure. When the vehicle is in motion, other limits apply: for forwardlooking systems the limit is $60 \mu \mathrm{W} / \mathrm{cm}^{2}$ at 3 $\mathrm{m}$ and for side-looking or rear-looking systems the limit is $30 \mu \mathrm{W} / \mathrm{cm}^{2}$ at $3 \mathrm{~m}$.

For the unlicensed systems at 59-64 GHz. power is limited to a density of $9 \mu \mathrm{W} / \mathrm{cm}^{2}$ at $3 \mathrm{~m}$.

There are no requirements for the modulation of any of these systems at this time. However, several of the commenting parties requested that the FCC establish a spectrum etiquette to minimize interference between users. Such a requirement was previously adopted for unlicensed Personal Communications Services (PCS) devices ${ }^{4}$ but has not been used in other contexts. While the Commission declined to set up a formal committee to develop such an etiquette, it did delay the effective date of the rules for a year and invite industry to submit a consensus proposal ${ }^{5}$.

Present rules allow amateur radio use of 76$77 \mathrm{GHz}$ at powers up to $1500 \mathrm{~W}$, although there is little or no use at present. In view of the safety applications of vehicular radars and the lack of knowledge of their susceptibility to interference, the Commission proposed suspending amateur use of this band pending a showing that interference is unlikely or the development of sharing criteria. To ensure amateur access to this section of spectrum, though, the Commission also proposed to upgrade the status of amateur use of 77.5-78 Ghz from secondary to co-primary. This would guarantee amateur access to a nearby band.

Discussions about the $76-77 \mathrm{GHz}$ vehicular radar band raised questions about the third harmonic of this band which lies in a radio astronomy band. Previously, the Commission had ruled out emission limits above $200 \mathrm{GHz}$. However, these new concerns lead the Commission to propose a new limit of $1000 \mathrm{pW} / \mathrm{cm}^{2}$ at $3 \mathrm{~m}$ for emissions in 200-231 GHz. This limit was based on tests between a prototype automotive radar and typical radio astronomy receivers. There is real concern that testing to show compliance with such a limit will be rather expensive and the Commission made clear that it is open to alternative methods to assuring that vehicular radar to radio astronomy interference does not occur.

\section{CONCLUSIONS}

Due to the regulated nature of radio spectrum, enabling regulations are needed for the opening of new bands. The FCC has 
taken a major step in opening the millimeter wave region for commercial use. It looks forward to extending these rules for other applications and working with other countries to harmonize international standards.

The views presented here are those of the author and not necessarily those of the FCC or US Government.

1. Notice of Proposed Rule Making, FCC Docket 94-124, 9 FCC Rcd 7078 (1994)

2. These comments are a matter of public record and are available for inspection in Washington at the FCC.

3. The text of the decision is available on World Wide Web at http://www.fcc.gov/Bureaus/Engineering_T echnology/Orders/fcc95499.txt

4. 47 C.F.R. 15.321-323

5. The Etiquette Working Group, a group of several firms discussing etiquette issues, maintains a World Wide Web page with information on their deliberations at http://www.atg.apple.com/areas/wireless/ 